\title{
Redox modulation of cellular stress response and lipoxin A4 expression by Hericium Erinaceus in rat brain: relevance to Alzheimer's disease pathogenesis
}

\author{
A. Trovato ${ }^{1 \dagger}$, R. Siracusa ${ }^{2+}$, R. Di Paola ${ }^{2}$, M. Scuto ${ }^{1}$, M. L. Ontario ${ }^{1}$, Ornella Bua ${ }^{1}$, Paola Di Mauro ${ }^{1}$, M. A. Toscano ${ }^{1}$, \\ C. C. T. Petralia', L. Maiolino', A. Serra', S. Cuzzocrea² and Vittorio Calabrese ${ }^{1 *}$
}

\begin{abstract}
Background: There has been a recent upsurge of interest in complementary medicine, especially dietary supplements and foods functional in delaying the onset of age-associated neurodegenerative diseases. Mushrooms have long been used in traditional medicine for thousands of years, being now increasingly recognized as antitumor, antioxidant, antiviral, antibacterial and hepatoprotective agent also capable to stimulate host immune responses.

Results: Here we provide evidence of neuroprotective action of Hericium Herinaceus when administered orally to rat. Expression of Lipoxin A4 (LXA4) was measured in different brain regions after oral administration of a biomass Hericium preparation, given for 3 month. LXA4 up-regulation was associated with an increased content of redox sensitive proteins involved in cellular stress response, such as Hsp72, Heme oxygenase -1 and Thioredoxin. In the brain of rats receiving Hericium, maximum induction of LXA4 was observed in cortex, and hippocampus followed by substantia Nigra, striatum and cerebellum. Increasing evidence supports the notion that oxidative stress-driven neuroinflammation is a fundamental cause in neurodegenerative diseases. As prominent intracellular redox system involved in neuroprotection, the vitagene system is emerging as a neurohormetic potential target for novel cytoprotective interventions. Vitagenes encode for cytoprotective heat shock proteins 70, heme oxygenase-1, thioredoxin and Lipoxin A4. Emerging interest is now focussing on molecules capable of activating the vitagene system as novel therapeutic target to minimize deleterious consequences associated with free radical-induced cell damage, such as in neurodegeneration. LXA4 is an emerging endogenous eicosanoid able to promote resolution of inflammation, acting as an endogenous "braking signal" in the inflammatory process. In addition, Hsp system is emerging as key pathway for modulation to prevent neuronal dysfunction, caused by protein misfolding.
\end{abstract}

Conclusions: Conceivably, activation of LXA4 signaling and modulation of stress responsive vitagene proteins could serve as a potential therapeutic target for AD-related inflammation and neurodegenerative damage.

Keywords: Lipoxin A4, Heat shock protein70, Heme Oxygenase-1, Nutritional mushrooms, Alzheimer's disease

\footnotetext{
* Correspondence: calabres@unict.it

${ }^{\dagger}$ Equal contributors

${ }^{1}$ Department of Biomedical and Biotechnological Sciences, School of

Medicine, University of Catania, Viale Andrea Doria 6, 95125 Catania, Italy

Full list of author information is available at the end of the article
}

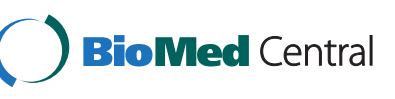

(c) 2016 The Author(s). Open Access This article is distributed under the terms of the Creative Commons Attribution 4.0 International License (http://creativecommons.org/licenses/by/4.0/), which permits unrestricted use, distribution, and reproduction in any medium, provided you give appropriate credit to the original author(s) and the source, provide a link to the Creative Commons license, and indicate if changes were made. The Creative Commons Public Domain Dedication waiver (http://creativecommons.org/publicdomain/zero/1.0/) applies to the data made available in this article, unless otherwise stated. 


\section{Background}

With the increased lifespan of the world's population, it is estimated that about 80 million people will suffer from dementia by 2040 whereby AD accounted for almost $60 \%$ of dementia cases [1-4]. Ageing, inexorable with decline in immune competence and onset of chronic inflammation, is one major causative factor associated to neurodegenerative diseases including dementia, Alzheimer's disease (AD) and Parkinson's disease (PD); atherosclerosis and stroke; diabetes; sarcopenia; and cancer [1-4]. Mushrooms have been used in traditional medicine for thousands of years $[5,6]$. Many controlled studies have since investigated the long list of medicinal actions thought to be associated with extracts of these and other mushrooms, including antitumor, immunomodulatory, antioxidant, antiviral, antibacterial, and hepatoprotective effects [7]. Mushrooms are a rich source of polysaccharides and low molecular weight peptides [8-11], and many have been shown to stimulate host immune responses [12, 13]. The administration of complex mixtures of molecules of unknown concentrations is difficult to reconcile with current pharmaceutical practices involving highly purified compounds. The active ingredients may be unknown, making mushroom extracts very difficult to patent. Moreover, mushroom-derived polysaccharides are complex molecules that cannot be synthesized, as the mass production of these compounds would require timely and costly extraction processes. As a result, many research efforts have focused on low molecular weight compounds, such as cordycepin [14]. Of the mushroom-derived therapeutics, polysaccharopeptides obtained from Agaricus campestris, Pleurotus ostreatus and Coriolus versicolor $[15,16]$, as well as Hericium erinaceus [17] are commercially the best established. While Coriolus versicolor is widely used to degrade recalcitrant organic pollutants such as pentachlorophenol (PCP) [18], H. erinaceus, has been used in traditional medical practices in China and Japan as to treat various human diseases. $H$. erinaceus mushroom is harvested, dried, ground, and made into medicinal preparations. The compounds isolated from fruiting bodies contain numerous biological activities, such as antitumor, hypolipidemic, hemagglutinating, cytotoxic, antimicrobial, endoplamic reticulum (ER) stress-suppressive, and antioxidant activities. In particular, it has been reported that hericenones and erinacines stimulate nerve growth factor (NGF) synthesis in cultured astrocytes. Erinacines have been isolated from cultured mycelia of $H$. erinaceus, and identified as one of diterpenoids. Hericenones have been isolated from the fruiting bodies of $H$. erinaceus and its molecular formula determined. In addition, studies from whole brain and cell cultures have shown that NGF affects the viability of cholinergic neurons, stimulating in the CNS the activity of enzymes, such as cholineacetyltransferase and acetylcholinesterase. These results are consistent with clinical finding indicating that $H$. erinaceus supplementation improves mild cognitive impairment, a condition where cholinergic neurons are centrally involved. However, despite the clinical relevance of $H$. erinaceus nutritional approaches, there have been only few studies elucidating mechanisms impacting defined brain physiopathological processes underlying the determinism of neurodegeneration involved in $\mathrm{AD}$, as well as other types of dementia.

Recently, the involvement of neuroinflammation and microglial activation in the pathogenesis of $\mathrm{AD}$ has been emphasized by compelling evidence from basic and clinical research studies indicating that inflammation induced by amyloid beta $(A \beta)$ is intimately associated with the development of AD neuropathology [19], in a scenario where $A \beta$ activates microglia [20], the resident macrophages of the brain, and activated microglia may then promote neuronal injury through the release of proinflammatory and cytotoxic factors, sustaining further glial activation via a detrimental cycle where neuroinflammation and oxidative stress act in synergy, exacerbating the course of the disease [21]. Consistent to this notion, recent advances in knowledge of the mechanisms of inflammatory resolution have identified lipoxins as attractive therapeutic tools to treat diseases in which inflammation is involved [22]. Lipoxin (LXA4) is generated via the lipoxygenase pathway during cell-cell interactions in inflammatory conditions, whereas aspirin-triggered LXA4 (ATL), a molecule that displays the same antiinflammatory activities as the native lipoxins, is generated after the acetylation of cyclooxygenase- 2 and is more resistant to metabolic inactivation [23]. However, the ability of LXA4 signaling to modulate neuroinflammation and AD pathology in vivo has not been yet completely elucidated. Recent data from our laboratory [24] have demonstrated that administration for one month of a biomass preparation from Coriolus versicolor was able to modulate redox-dependently cellular stress response in rat. In view of the neuroinflammatory nature of Alzheimer's disease pathogenesis and taken into account the therapeutic potential of mushroom nutritional approaches, the present study was conducted in order to gain insight into the possible neuroprotective role of $\mathrm{H}$. erinaceus biomass preparation against the inflammatory process and to evaluate the impact of this intervention on cellular stress response mechanism operating in rat brain.

\section{Methods}

\section{Chemicals}

All reagents were from Merck (Germany) and of the highest grade available. Hericium erinaceus powder containing both mycelium and primordia (young fruit body) was supplied by Mycology Research Laboratories Ltd. (United Kingdom). 


\section{H. erinaceus biomass preparation}

$H$. erinaceus is found almost worldwide; however, its bioactivity varies depending on the habitat in which it grows. To eliminate these variations in the present study an established $H$. erinaceus strain was used which demonstrates rapid and aggressive colonization. Our H. erinaceus powder containing both mycelium and primordia (young fruit body) was supplied by Mycology Research Laboratories Ltd. This mushroom, cultivated into a biomass, is grown on a sterilized (autoclaved) substrate. The production process involves the inoculation of sterile organic edible grain with spawn from the mother culture. The fungus is thus allowed to colonize completely in an environment where the growth medium is aseptically kept. At the correct stage of development, corresponding to the maximum bioavailability the living biomass is aseptically air-dried, granulated, tested microbiologically and reduced in powder. In comparison to $H$. erinaceus extracts, biomass has the advantage of preserving all nutraceutical potential which is usually reduced with extracts or concentrates, including lyophilisation, and thus the activity of the product corresponds with the source mushroom, while being further intensified by utilizing the entire mycelium. The $H$. erinaceus biomass powder containing mycelium and primordia of the respective mushroom was used for experiments.

\section{Animals}

The study was carried out using male Sprague-Dawley rats (200 to 230 g; Harlan, Nossan, Italy). Food and water were available ad libitum. The study was approved by the University of Messina Review Board for the care of animals. Animal care was in compliance with Italian regulations on the protection of animals used for experimental and other scientific purposes (DM116192) as well as with the relevant European Economic Community (EEC) regulations (OJ of EC L 358/1 12/18/1986).

\section{Experimental groups and treatments}

Animals were randomly allocated into the following groups:

Group 1: Sham + Veh = vehicle solution (saline) was daily administered o.s. for 3 month $(N=10)$; Group 2: Sham $+H$. erinaceus $=$ same as the Sham + Veh group, but $H$. erinaceus powder $(200 \mathrm{mg} / \mathrm{kg}$ body weight, soluble in saline o.s.) was administered daily by stomach gavage for the full experiment duration of 3 month $(N=10)$.

At the proper time points, animals were killed, brains quickly removed and dissected into the cerebral cortex, hippocampus, striatum and cerebellum, according to a standardized procedure, in a cold anatomical chamber and following a protocol that allows a maximum of $50 \mathrm{~s}$ time-variability for each sample across animals. SN was dissected from the deepest part of the interpeduncolar fossa. Samples from different rat brain areas, total brain, and from liver and kidney were homogenized for $2 \mathrm{~min}$ in $0.05 \mathrm{~mol} / \mathrm{L}$ Tris-HCl buffer, pH 7.4 (1:9).

\section{Sampling and lymphocyte purification}

Blood was collected through cardiac puncture and added to tubes containing EDTA. Aliquots $(2 \mathrm{ml})$ were utilized for lymphocytes purification, which was accomplished by using the Ficoll Paque System following the procedure provided by the manufacturer (GEHealthcare, Piscataway, NJ, USA).

\section{Western blot analysis}

Inducible heat shock protein 70 (Hsp-70), heme oxygenase-1 (HO-1) and thioredoxin (Trx) protein levels were estimated by Western blot analysis which was accomplished as previously described [25]. Plasma samples were processed as such, while the isolated lymphocyte pellet as well as dissected brain regions brain were homogenized and centrifuged at $10,000 \times g$ for $10 \mathrm{~min}$. The supernatant was then used for analysis after determination of protein content. Proteins extracted for each sample, at equal concentration $(50 \mu \mathrm{g})$ were boiled for $3 \mathrm{~min}$ in sample buffer (containing $40 \mathrm{mM}$ Tris- $\mathrm{HCl}$ pH7.4, $2.5 \%$ SDS, 5 \% 2mercaptoethanol, $5 \%$ glycerol, $0.025 \mathrm{mg} / \mathrm{ml}$ of bromophenol blue) and then separated on a polyacrylamide mini gels precasting 4-20\% (codNB10420 NuSept Ltd Australia). Separated proteins were transferred onto nitrocellulose membrane (BIO-RAD, Hercules, CA,USA) in transfer buffer containing (0.05 \% SDS, 25 mM Tris, $192 \mathrm{mM}$ glycine and $20 \% \mathrm{v} / \mathrm{v}$ methanol). The transfer of the proteins on the nitrocellulose membrane was confirmed by staining with Ponceau Red which was then removed by three washes in PBS (phosphate buffered saline) for $5 \mathrm{~min}$ each. Membranes were then incubated for $1 \mathrm{~h}$ at room temperature in $20 \mathrm{mM}$ Tris $\mathrm{pH} 7.4,150 \mathrm{mM} \mathrm{NaCl}$ and Tween 20 (TBS-T) containing $2 \%$ milk powder and incubated with appropriate primary anti-HSP-72, anti- HO-1, and anti-Trx polyclonal antibodies (Santa Cruz Biotech. Inc.), overnight at $4{ }^{\circ} \mathrm{C}$ in TBS-T. The same membrane was incubated with a goat polyclonal antibody anti-beta-actin (SC 1615 Santa Cruz Biotech.Inc., Santa Cruz, CA, USA, dilution $1: 1000)$ to verify that the concentration of protein loaded in the gel was the same in each sample. Excess unbound antibodies were removed by three washes are with TBS-T for $5 \mathrm{~min}$. After incubation with primary antibody, the membranes were washed three times for $5 \mathrm{~min}$. in TBS-T and then incubated for $1 \mathrm{~h}$ at room temperature with the secondary polyclonal antibody conjugated with horseradish peroxidase (dilution1:500). The membranes were then washed three times with TBS-T for $5 \mathrm{~min}$. Finally, the 
membranes were incubated for 3 min with Super Signal chemiluminiscence detection system kit (Cod34080 Pierce Chemical Co, Rockford, IL, USA) to display the specific protein bands for each antibody. The immunoreactive bands were quantified by capturing the luminescence signal emitted from the membranes with the Gel Logic 2200 PRO (Bioscience) and analyzed with Molecular Imaging software for the complete analysis of regions of interest for measuring expression ratios. The molecular weight of proteins analyzed was determined using a standard curve prepared with protein molecular weight.

\section{Quantification of LXA4}

LXA4 was analyzed using ELISA kit (CEB452Ge Cloud-Clone Corp). The assay was performed following the instructions of the manufacturer and measured by a microplate reader, by reading at $450 \mathrm{~nm}$. For this assay, aliquots of plasma or tissues homogenates were used. Tissue samples after rinsed in to remove excess blood thoroughly and weighed before homogenization, were homogenized in ice-cold PBS (0.01 M, pH 7.2). The resulting suspension was sonicated and then centrifuged for 5 min at $5000 \times \mathrm{g}$ at $4{ }^{\circ} \mathrm{C}$, and supernatants collected. Aliquots of standard, blank, plasma or tissue homogenates containing equal amounts of proteins were brought to a final volume of $50 \mu \mathrm{L}$ and added into the appropriate wells, followed by addition of $50 \mu \mathrm{L}$ of detection reagent A to each well. The plate was shaken gently before incubation for $1 \mathrm{~h}$ at $37^{\circ} \mathrm{C}$. The solution was aspirated and the wells were washed 3 times. The remaining liquid was removed completely by snapping the plate onto absorbent paper. $100 \mu \mathrm{L}$ of detection reagent $\mathrm{B}$ were then added to each well followed by incubation for $30 \mathrm{~min}$ at $37^{\circ} \mathrm{C}$. After repeated washing (5 times), $90 \mu \mathrm{L}$ of substrate solution was added to each well followed by incubation for $25 \mathrm{~min}$ at $37^{\circ} \mathrm{C}$. Finally, $50 \mu \mathrm{L}$ of stop solution was added to each well, before measurement through reading at $450 \mathrm{~nm}$.

\section{Statistical analysis}

All results obtained were expressed in mean \pm SEM. The data was analyzed by ANOVA to compare the different groups and considered significant with a $p<0.05$. Each experiment was carried out in triplicate and repeated twice.

\section{Results}

Regional distribution of LXA4 expression in rat brain after treatment with $\mathrm{H}$. erinaceus

Expression of LXA4, in control animals and after administration of $H$. erinaceus biomass preparation, was investigated in different brain regions of rats, as well as in plasma and peripheral tissues, such as liver and kidney. Administration of $H$. erinaceus for 3 month at the oral daily dose of $200 \mathrm{mg} / \mathrm{Kg}$ induced an increase in the protein level of LXA4 in all brain regions examined. As shown in Fig. 1, highest levels of Hericium-dependent increase of LXA4 protein expression were observed in cortex, hippocampus, as well as in total brain, followed with a statistically significant difference by substantia nigra, striatum, and cerebellum.

\section{Mushroom-induced increase of LXA4 in peripheral tissues} In the group of animals receiving chronic administration of $H$. erinaceus, compared to untreated controls, brain changes in LXA4 protein were associated with a significant $(p<0.05)$ increase in the plasma and in lymphocytes (Fig. 2a), as well as in peripheral organs, such as liver and kidney, as illustrated in Fig. 2b.

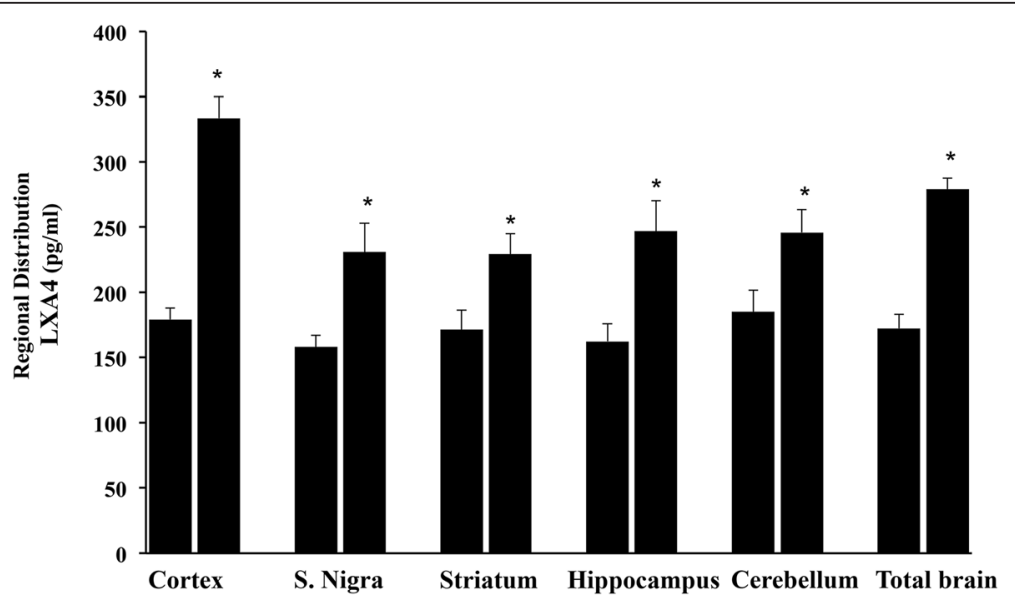

Fig. 1 Regional distribution of Lipoxin A4 in rat brain. LXA4 protein levels in different brain regions and in total brain of control or $\mathrm{H}$. erinaceus-fed rats. Values are expressed as mean \pm SEM of three independent analyses on 10 animals per group. CX: cortex; Hp: Hippocampus; Cb: cerebellum; TB: Total Brain. H. erinaceus, given orally at the dose of $200 \mathrm{mg} / \mathrm{Kg}$ for 3 month. ${ }^{*} P<0.05$ vs control 


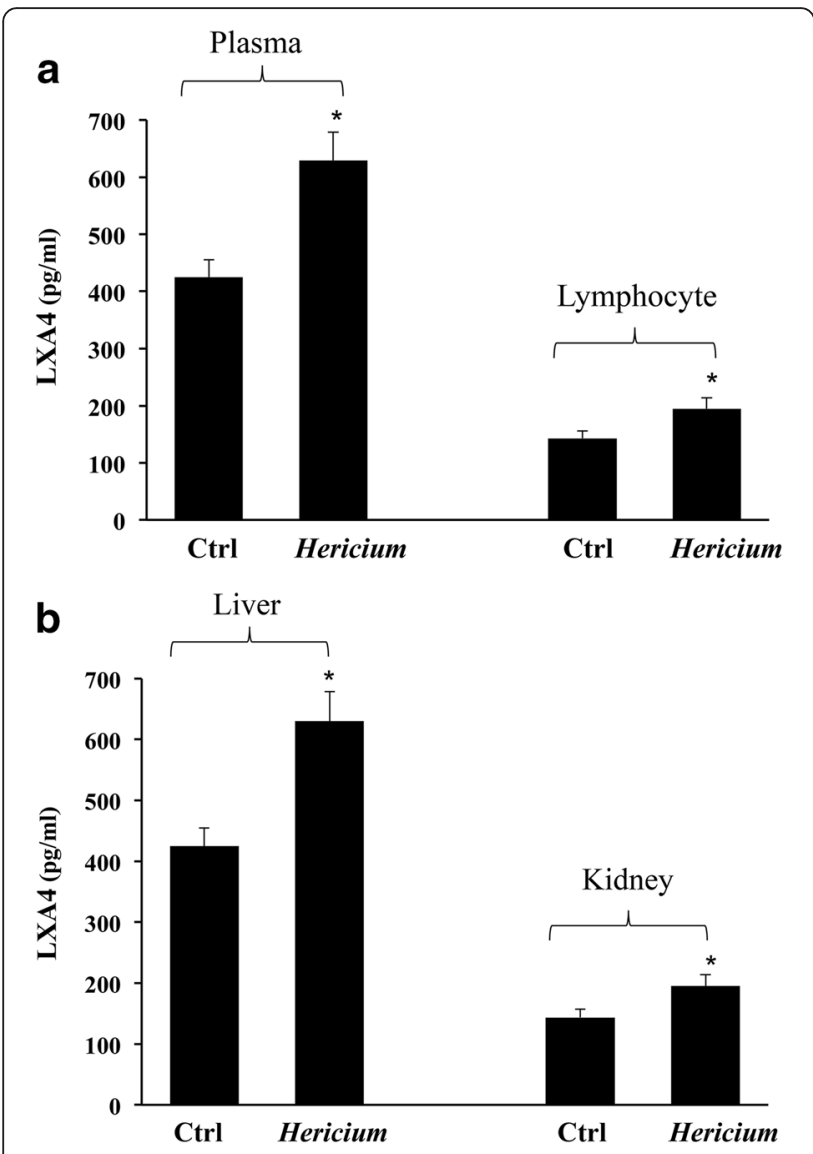

Fig. 2 a Lipoxin A4 in rat blood. LXA4 levels in plasma and in lymphocytes from rats fed $H$. erinaceus biomass preparation as compared to control group. Data are expressed as mean \pm SEM of 10 animals per group. ${ }^{*} P<0.05$ vs controls. b Lipoxin A4 in Liver and Kidney. LXA4 levels in liver and kidney from rats fed $H$. erinaceus biomass preparation as compared to control group. Data are expressed as mean \pm SEM of 10 animals per group. ${ }^{*} P<0.05$ vs controls

Modulation of HO-1, Hsp70 and Thioredoxin protein expression in rat brain after mushroom supplementation As reported in Fig. 3a and b, mushroom supplementation with $H$. erinaceus biomass preparation, resulted in upregulation of brain cellular stress response proteins $\mathrm{HO}-1$, and the inducible isoform of Hsp70. A representative Western blot, obtained probing total brain tissue homogenate with an antibody specific for the inducible isoform of heme oxygenase protein, is also shown (Fig. 3a). Western blot analysis of the inducible heat shock proteins 70 (Hsp72) revealed a significant increase in the brain of animals receiving $H$. erinaceus compared to control group. In the same figure a representative blot is reported (Fig. 3b). Figure 3c reports results from analysis of Trx protein expression in total brain of animals receiving $H$. erinaceus, as compared to controls. As shown, Trx protein increases significantly after Hericium supplementation. These results were also confirmed when measuring HO-1, Hsp70 and Trx protein expression in different brain regions of animals supplemented with $H$. erinaceus, as compared to the control group (Fig. $4 \mathrm{a}-\mathrm{c}$ ). Figure $4 \mathrm{a}$ shows a significant increase of $\mathrm{HO}-1$ protein levels induced by this nutritional mushroom in the cortex and in the hippocampus, whereas this increase was not statistically significant $v s$ controls in the cerebellum. In the same figure a representative Western blot, obtained probing the different brain regions for $\mathrm{HO}-1$ is reported (Fig. 4a).

Figure $4 \mathrm{~b}$ shows increased Hsp70 protein expression after $H$. erinaceus administration, which was significant, compared to control animals, in the cortex, hippocampus and cerebellum. In the same figure a representative Western blot, obtained probing the different brain regions for inducible isoform of $\mathrm{Hsp} 70$ is reported (Fig. 4b). Consistent with HO-1 and Hsp70 data, analysis of thioredoxin protein revealed that $H$. erinaceus biomass supplementation upregulated this protein levels in the brain regions of cortex, hippocampus and cerebellum (Fig. 4c), which was statistically significant in all brain regions examined $(P<0.05$ vs control).

We then investigated whether administration of $H$. erinaceus would have affected systemic as well as peripheral stress response. As illustrated in Fig. 5a a significant induction of HO-1 protein was observed in the plasma, lymphocytes and, respectively, liver and kidney of $H$. erinaceus fed rats, as compared to untreated rat group. Representative blots are reported in the same figure. Figure $5 \mathrm{~b}$ shows a parallel increase in Hsp70 protein expression, which was found in plasma, lymphocytes and liver, but not in the kidney of $H$. erinaceus fed animals. Consistent to the notion that mushroom biomass supplementation modulates stress responsive vitagenes we also found an increased protein expression of the redox sensitive Trx in lymphocyte of $H$. erinaceus fed rat, which was significantly higher respect to basal expression of this redox protein measured in control, untreated animals (Fig. 6). A representative Western blot, obtained probing total brain tissue homogenate with an antibody specific for the Trx protein is shown in the same figure.

\section{Discussion}

$\mathrm{AD}$ has gained widespread attention because of its high economic costs, which have reached $\$ 400$ billion per year in the USA alone, as well as the social costs, which are more difficult to quantify [26]. AD-related pathological markers include a progressive death of neurons in specific areas with an accumulation of intracellular neurofibrillary tangles (NFTs) and extracellular depositions of amyloid plaques (APs). NFTs are composed of the misfolded hyperphosphorylated microtubule-associated protein Tau (MAPT or Tau), whereas APs are extracellular deposits of misfolded and aggregated amyloid-beta peptides $(A \beta)[27,28]$. Because both NFTs and APs are persistently found in areas with severe neuronal 


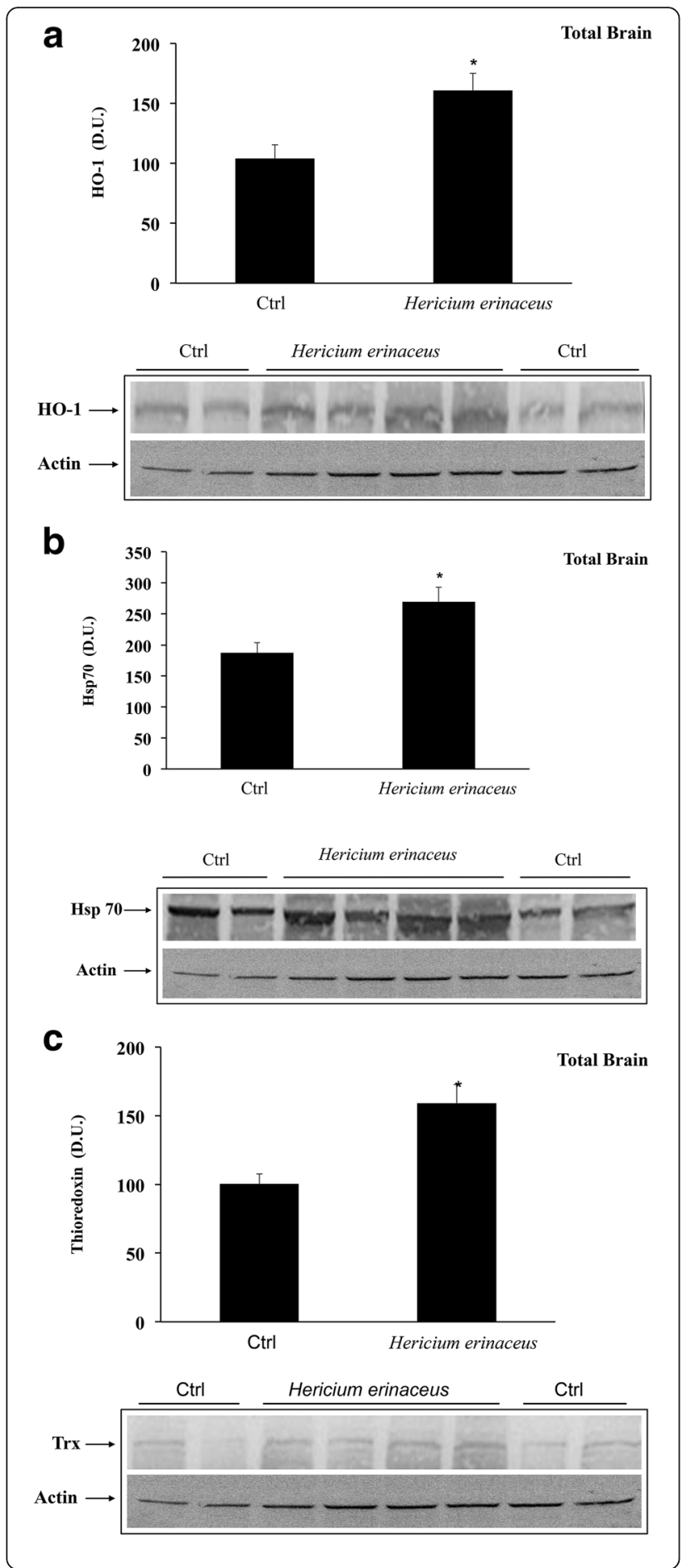

Fig. 3 a Inducible heme oxygenase in Total Brain. HO-1 protein levels in the brain of rats fed $\mathrm{H}$. erinaceus biomass preparation as compared to control group. Total brain homogenates from control and mushroom supplemented rats were assayed for HO-1 expression by Western blot. The bar graph shows densitometric values, expressed as mean standard error of mean of 3 independent analyses. $P<0.05$ vs control. In the representative immunoblot shown, $\beta$-actin has been used as loading control. D.U., densitometric units. b Inducible Heat shock protein 70 in Total brain. Hsp70 protein levels in the brain of rats fed $\mathrm{H}$. erinaceus biomass preparation as compared to control group. Total brain homogenates from control and mushroom supplemented rats were assayed for Hsp70 expression by Western blot. The bar graph shows densitometric values, expressed as mean standard error of mean of 3 independent analyses. $P<0.05$ vs control. In the representative immunoblot shown, $\beta$-actin has been used as loading control. D.U., densitometric units. c Thioredoxin in Total brain. Trx protein levels in the brain of rats fed $H$. erinaceus biomass preparation as compared to control group. Total brain homogenates from control and mushroom supplemented rats were assayed for Trx expression by Western blot. The bar graph shows densitometric values, expressed as mean standard error of mean of 3 independent analyses. $P<0.05$ vs control. In the representative immunoblot shown, $\beta$-actin has been used as loading control. D.U., densitometric units

death, these proteins were considered to be the main cause of neuronal loss and the emergence of dementia, which is a crucial symptom of AD; however, numerous drug trials based on these proteins have failed to provide a useful $A D$ therapy [28]. A post-mortem study demonstrated that the misfolded protein accumulation is a shared pattern in many neurodegenerative diseases, including AD [25], concurring to the conclusion that accumulation of misfolded proteins is a prominent potential cause of neurodegeneration in AD [29]. Recently, the involvement of neuroinflammation and microglial activation in the pathogenesis of $\mathrm{AD}$ has been emphasized by compelling evidence from basic and clinical research studies indicating that inflammation induced by $\mathrm{A} \beta$ is intimately associated with the development of AD neuropathology [19]. Relevant to the central role of neuroinflammation in $\mathrm{AD}$ pathgenesis, are recent advances in knowledge of the mechanisms of inflammatory resolution, identifying lipoxins as attractive therapeutic tools to treat diseases in which inflammation is involved [22]. LXA4 is generated via the lipoxygenase pathway during cell-cell interactions in inflammatory conditions, whereas aspirin-triggered LXA4 (ATL), a molecule that displays the same anti-inflammatory activities as the native lipoxins, is generated after the acetylation of cyclooxygenase- 2 and is more resistant to metabolic inactivation [23]. Lipoxins potentiate inflammatory resolution by means of potent agonistic actions at the G-protein coupled receptor, termed LXA4 receptor (ALX/FPR2). Activation of ALX by LXA4 reduces many endogenous processes, such as neutrophil and eosinophil recruitment and activation, leukocyte migration, NF-kB translocation, and chemokine and cytokine production [22]. Likewise, evidence shows that LXA4 signaling primes 


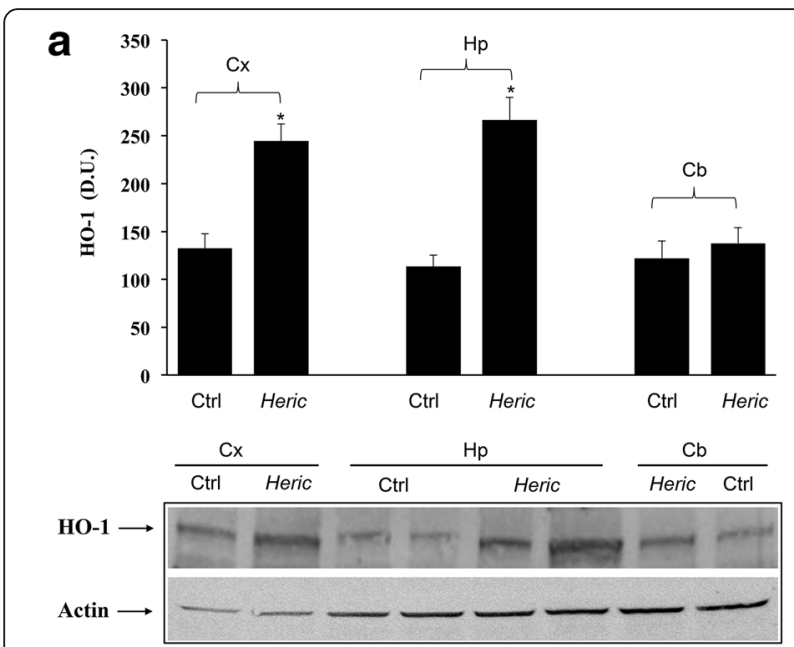

b
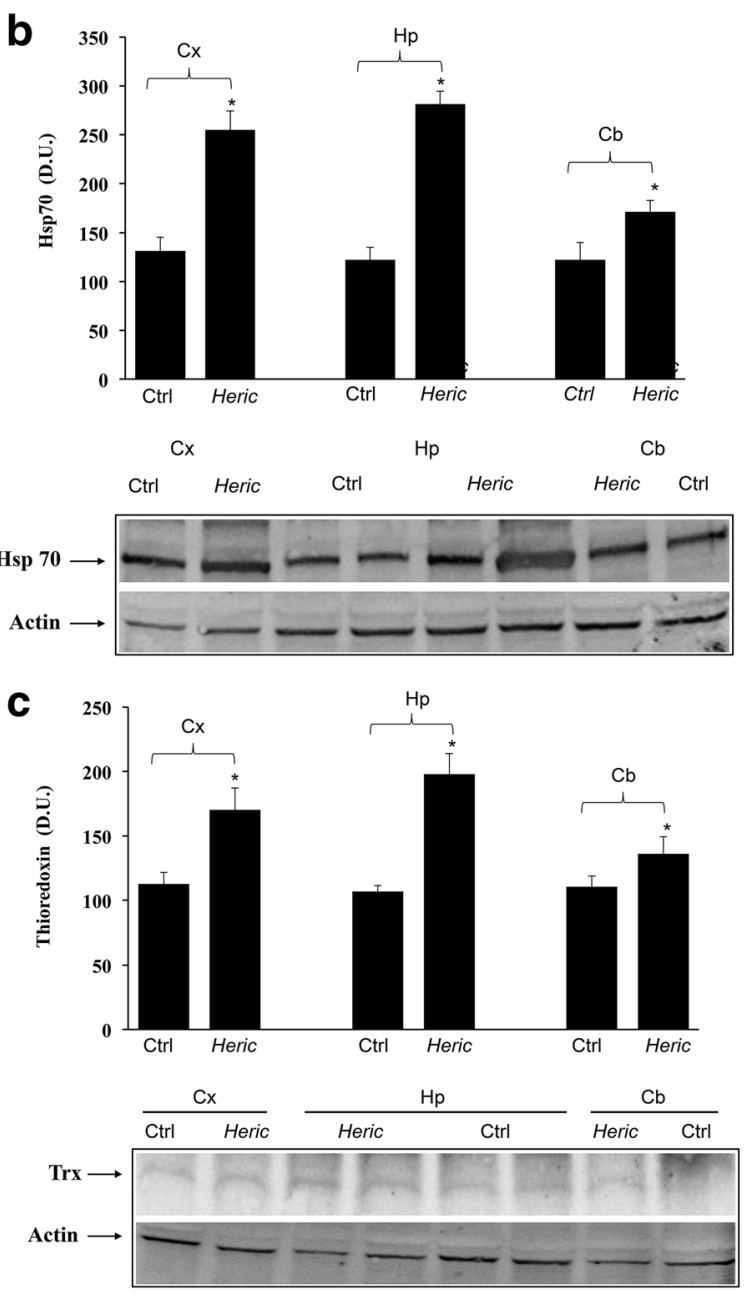

Fig. 4 a Regional distribution of $\mathrm{HO}-1$ in rat brain. $\mathrm{HO}-1$ protein levels in different brain regions of rats fed $\mathrm{H}$. erinaceus biomass preparation as compared to control group. Brain region homogenates from control and mushroom supplemented rats were assayed for $\mathrm{HO}-1$ expression by Western blot. The bar graph shows densitometric values, expressed as mean standard error of mean of 3 independent analyses. $P<0.05$ vs control. In the representative immunoblot shown, $\beta$-actin has been used as loading control. D.U., densitometric units. CX: cortex; Hp: Hippocampus; Cb: cerebellum. b Regional distribution of Hsp70 in rat brain. Inducible Hsp70 protein levels in different brain regions of rats fed $\mathrm{H}$. erinaceus biomass preparation as compared to control group. Brain region homogenates from control and mushroom supplemented rats were assayed for Hsp70 expression by Western blot. The bar graph shows densitometric values, expressed as mean standard error of mean of 3 independent analyses. $P<0.05$ vs control. In the representative immunoblot shown, $\beta$-actin has been used as loading control. D.U., densitometric units. CX: cortex; Hp: Hippocampus; Cb: cerebellum. c Regional distribution of thioredoxin in rat brain. Trx protein levels in different brain regions of rats fed $H$. erinaceus biomass preparation as compared to control group. Brain region homogenates from control and mushroom supplemented rats were assayed for Trx expression by Western blot. The bar graph shows densitometric values, expressed as mean standard error of mean of 3 independent analyses. $P<0.05$ vs control. In the representative immunoblot shown, $\beta$-actin has been used as loading control. D.U., densitometric units. CX: cortex; Hp: Hippocampus; Cb: cerebellum

macrophages for chemotaxis and enhances phagocytosis of microorganisms and apoptotic cells. In the nervous system, LXA4 protects neurons against experimental stroke and $A \beta_{42}$ toxicity by modulating inflammation. In addition, lipoxins inhibit inflammatory pain processing through their actions on astrocytic activation in the spinal cord $[30,31]$. However, the ability of LXA4 signaling to modulate neuroinflammation and AD pathology in vivo has not been yet completely elucidated.

Mushrooms provide a great potential as a polypharmaceutic drug because of the complexity of their chemical contents and different varieties of bioactivities. If available evidence suggests anti-oxidants, anti-tumor, antivirus, anti-cancer, anti-inflammatory, immune modulating, antimicrobial, and anti-diabetic activities from mushrooms [32], however, contrarily to plant herbal medicines, which are widely explored and relatively more advanced, the brain and cognition health effects of mushrooms are in the early stages of research. Here, by extending previous finding on nutritional approaches to neuroinflammation, we provide experimental evidence that administration of $H$. erinaceus for 3 month to rats results in up regulation of vitagenes, in particular Hsp70, HO-1 and Trx, an effect associated with increased synthesis of LXA4 in different brain regions of rat. This latter, an endogenous eicosanoid, is emerging as an important resolvin, a class of compounds endowed with the capability to promote resolution of inflammation, therefore suggesting that nutritional modulation with $H$. erinaceus, through redoxdependent vitagene network might activate endogenous 


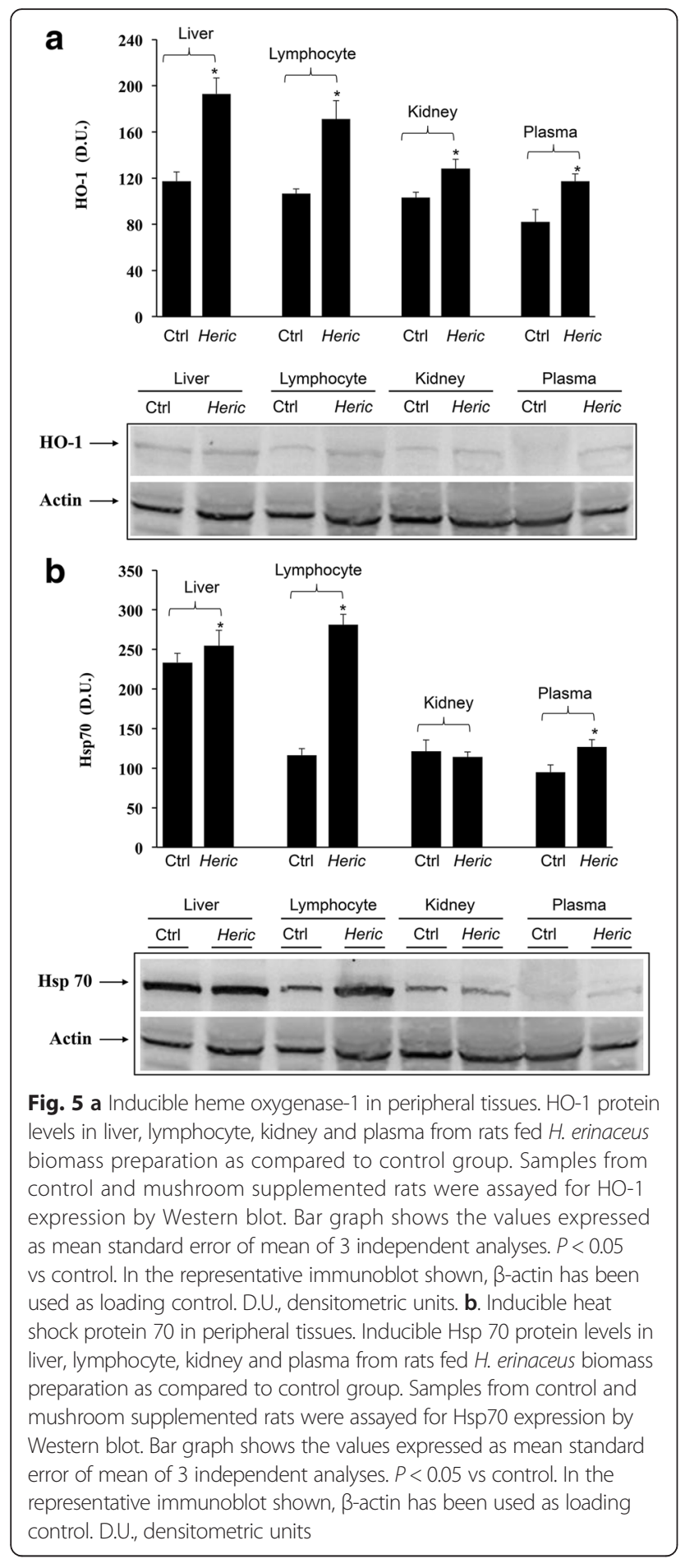

"braking signal" processes impacting the inflammatory process. We also provide evidence of neuroprotective action of $H$. erinaceus when administered orally to rat. Expression of LXA4, measured in different brain regions after oral administration of a biomass $H$. erinaceus preparation for 3 month increased significantly in all brain regions examined, as compared to control group of animals, particularly in cortex and cerebellum, followed by substantia Nigra, striatum and cerebellum. LXA4 upregulation was associated with an increased content of redox sensitive proteins involved in cellular stress response, such as Hsp72, HO-1 and Trx. We show that SN exhibited lower LXA4 content respect to other brain regions examined, both in control and mushroom stimulated animals. This finding is relevant to $\mathrm{AD}$ and $\mathrm{PD}$ pathogenesis, particularly to theories connecting aging and neuronal degeneration with oxidative damage. SN neurons are depleted during physiological aging and even more so in all neurodegenerative processes associated with Parkinsonian symptoms. [33-37]. In addition, we demonstrate that $H$. erinaceus treatment resulted in a significant increase of LXA4 in most of the brain regions examined and modulated expression of cytoprotective proteins, such as HO-1, Hsp70 and Trx. Our results are consistent with recent evidence obtained in mice, showing neuroprotection by $H$. erinaceus on Ab25-35 peptide-induced cognitive dysfunction $[38,39]$. In this study the powder of $H$. erinaceus was mixed with a normal powdered diet and the Ab25-35 peptide was administered by intracerebroventricular injection. The results revealed that $H$. erinaceus prevented impairments of spatial short-term and visual recognition memory induced by Ab25-35 in mice. Furthermore, human trials with $H$. erinaceum derivatives also have showed promising results in patients with dementia based on Revised Hasegawa Dementia Scale (HDS-R) [40].

Our results indicating that nutritional modulation of critical proteins involved in brain stress tolerance can be achieved via supplementation with a well characterized strain of $H$. erinaceus are relevant to those theories connecting proteome control quality failure with ageassociated neurodegenerative diseases. Consistent to this notion, in AD pathology, the accumulation of APs composed of $\mathrm{A} \beta$ aggregates and neurofibrillary tangles NFTs composed of misfolded Tau proteins, accumulation of these proteins as consequence of faulty protein quality control mechanisms, is associated with a deficit in those mechanisms participating to induction of cytoprotective proteins (Hsps) or, more in general, involved in the cellular pathways of stress tolerance. It is conceivable that in these conditions administration of $H$. erinaceus mushroom, which increases the redox potential associated with induction of vitagenes, may help vulnerable neurons to resist to proteotoxic insults and hence to apoptotic neurodegeneration. This is furtherly corroborated by the finding indicating that restoration of normal proteostasis is crucial for neuronal survival [41].

The molecular chaperone Hsp70 protects cells from injury by binding damaged proteins under stressful situations. Members of the $70 \mathrm{kDa}$-heat shock protein family (Hsp70s) are, in their function as molecular chaperones, 


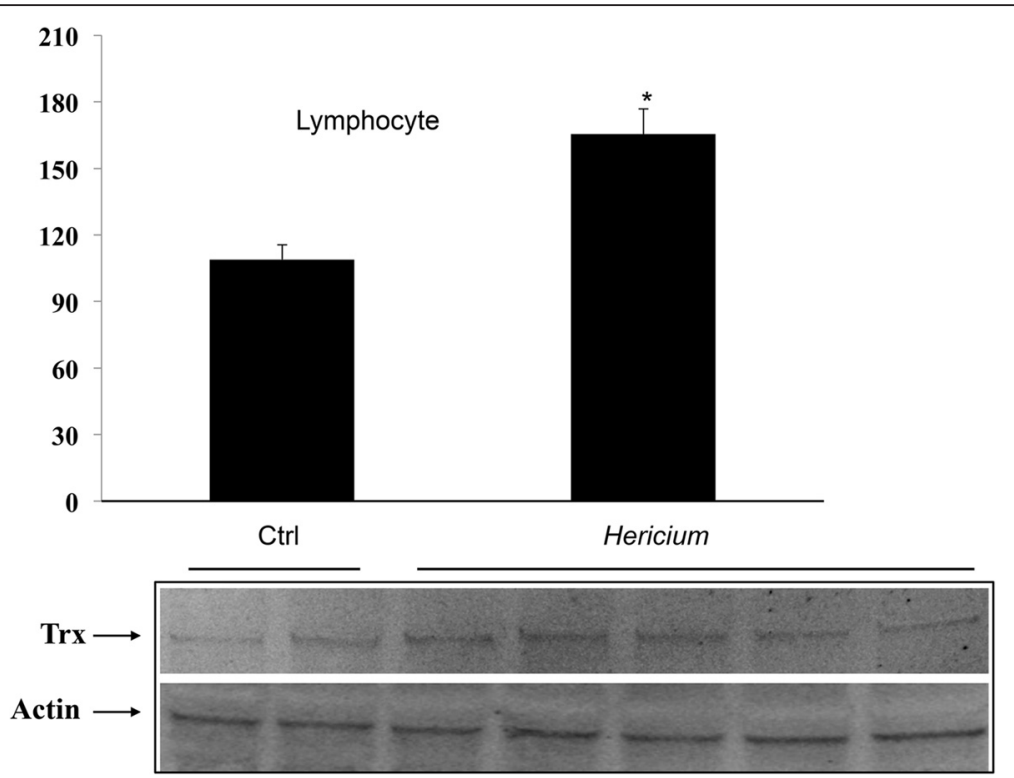

Fig. 6 Inducible Thioredoxin in peripheral tissues. Trx protein levels in lymphocyte from rats fed H. erinaceus biomass preparation as compared to control group. Samples from control and mushroom supplemented rats were assayed for Trx expression by Western blot. Bar graph shows values expressed as mean standard error of mean of 3 independent analyses. $P<0.05$ vs control. The representative immunoblot shows Trx and $\beta$-actin, which has been used as loading control. D.U., densitometric units

involved in folding of newly synthesized proteins and refolding of damaged or misfolded proteins, as well as in assembly and disassembly of protein complexes. All human Hsp70s have highly conserved domain structures [42]. They consist of an N-terminal ATPase domain, a middle region and an N-terminal peptide binding domain. However, they differ in expression patterns, cellular localization and function. There are Hsp70's specifically located in the endoplasmatic reticulum (Grp78, also known as BiP) and in the mitochondria (Grp75, also known as mortalin). However, the members which are mainly located in the cytosol and nucleus are the heat shock cognate protein 70 (Hsc70) and the heat shock protein 70 (Hsp70). Cellular stress often leads to protein unfolding and, therefore, to increased protein hydrophobicity, which may result in the formation of toxic protein aggregates [42]. As recently demonstrated, Hsp70 expression is induced under the mild oxidative stress conditions, when oxidative damage to proteins leads to their unfolding [43], and the heat shock response is activated driving increases in the expression of molecular chaperones, which reaches about two-fold the baseline levels [43]. Although HSP's can refold mildly disordered proteins, it is clear that HSP's are not able to repair covalently-modified oxidized proteins or to reverse oxidative protein modifications, which results in increased protein hydrophobicity, as triggering signal for the activation of a highly regulated and rapid series of events, called the 'heat shock response' (HSR). Heat shock transcription factor 1 (HSF1) is bound to a complex of heat shock proteins (Hsps), such as Hsp70 and Hsp90, during non- stressed conditions and, therefore, kept in an inactive state. When Hsps recognize hydrophobic patches of damaged and unfolded proteins, the Hsps dissolve from the complex with HSF1 in response to cellular stress. This event is followed by HSF1 trimer formation, which further leads to the activation and translocation of the transcription factor into the nucleus, where the trimer binds to the heat shock gene promoters, the so called heat shock elements (HSEs). This leads to the fast expression of Hsps [44]. Moreover, the heat shock genes do not contain introns, which further accelerates their expression. An unfolded protein that binds to Hsp70 may be either refolded into its native nontoxic conformation and then released, or may stay bound by Hsp70 to protect non-damaged molecules. Since most oxidative protein modifications are not repairable due to their covalent nature, the majority of oxidized proteins are degraded by the proteasomal system.

\section{Conclusions}

Our finding can open up new neuroprotective strategies, as interventions aiming at inducing the vitagene defense system mechanism, including Hsps, HO-1, thioredoxin and lipoxin $\mathrm{A} 4$, can represent a therapeutic target to minimize the deleterious consequences associated to oxidative stress, such as in brain aging and neurodegenerative disorders.

\section{Abbreviations}

$A D$, Alzheimer's disease; APs, amyloid plaques; ATL, aspirin-triggered LXA4; AB, amyloid-beta peptides; EEC, European Economic Community; ER, endoplamic reticulum; H. erinaceus, Hericium. erinaceus; HDS-R, Revised Hasegawa Dementia 
Scale; HO-1, heme oxygenase-1; Hsc70, heat shock cognate protein 70; HSEs, heat shock elements; HSF1, Heat shock transcription factor 1; Hsp-70, heat shock protein 70; HSR, heat shock response; LXA4, Lipoxin A4; MAPT or Tau, microtubule-associated protein Tau; NFTs, intracellular neurofibrillary tangles; NGF, nerve growth factor; PCP, pentachlorophenol; PD, Parkinson's disease; St, striatum; Trx, thioredoxin

\section{Acknowledgements}

Authors acknowledge T. Fernandes for helpful discussions.

\section{Authors' contributions}

AT participated in study design, LXA4 protein determination and experimental western blot analysis, writing of methods section and contributes to manuscript drafting. RS participated in study design, animal treatments and experimental western blot analysis and contributes to manuscript drafting. RD participated in study design, animal treatments and experimental western blot analysis and contributes to manuscript drafting. MS, MLO, PD participated in study design, methods section writing and western blot analysis. MAT, CCTP MO participated in study design, drafting of manuscript and reference updating. LM participated in study design and drafted the manuscript. AS participated in study design and drafted the manuscript. SC and VC conceived the study, participating in its design and coordination and helped to draft the manuscript. All authors read and approved the final manuscript.

\section{Competing interests}

The authors declare that they have no competing interests.

\section{Ethics approval and consent to participate}

The study was approved by the University of Messina Review Board for the care of animals. Animal care was in compliance with Italian regulations on the protection of animals used for experimental and other scientific purposes (DM116192) as well as with the relevant European Economic Community (EEC) regulations (OJ of EC L 358/1 12/18/1986).

\section{Author details}

${ }^{1}$ Department of Biomedical and Biotechnological Sciences, School of Medicine, University of Catania, Viale Andrea Doria 6, 95125 Catania, Italy. ${ }^{2}$ Department of Chemical, Biological, Pharmaceutical and Environmental Sciences, University of Messina, Messina, Italy.

\section{Received: 1 April 2016 Accepted: 1 July 2016}

\section{Published online: 09 July 2016}

\section{References}

1. Di Bona D, Accardi G, Virruso C, Candore G, Caruso C. Association between genetic variations in the insulin/insulin-like growth factor (Igf-1) signaling pathway and longevity: a systematic review and meta-analysis. Curr Vasc Pharmacol. 2014;12(5):674-81.

2. Candore G, Balistreri CR, Listi F, Grimaldi MP, Vasto S, et al. Immunogenetics, gender, and longevity. Ann N Y Acad Sci. 2006;1089:516-37.

3. Martorana A, Bulati M, Buffa S, Pellicano M, Caruso C, et al. Immunosenescence, inflammation and Alzheimer's disease. Longev Healthspan. 2012;1:8.

4. Calabrese EJ, Dhawan G, Kapoor R, lavicoli I, Calabrese V. What is hormesis and its relevance to healthy aging and longevity? Biogerontology. 2015; 16(6):693-707.

5. Elsayed EA, El Enshasy H, Wadaan MA, Aziz R. Mushrooms: a potential natural source of anti-inflammatory compounds for medical applications. Mediators Inflamm. 2014;2014:805841.

6. El Enshasy H, Elsayed EA, Aziz R, Wadaan MA. Mushrooms and truffles: historical biofactories for complementary medicine in Africa and in the middle East. Evid Based Complement Alternat Med. 2013;2013:620451.

7. Paterson RR, Lima N. Biomedical effects of mushrooms with emphasis on pure compounds. Biomed J. 2014;37(6):357-68.

8. Komura DL, Ruthes AC, Carbonero ER, Gorin PA, lacomini M. Water-soluble polysaccharides from Pleurotus ostreatus var. florida mycelial biomass. Int J Biol Macromol. 2014;70:354-9.

9. Wasser SP. Medicinal mushroom science: Current perspectives, advances, evidences, and challenges. Biomed J. 2014;37(6):345-56.

10. Lindequist U, Kim HW, Tiralongo E, Van Griensven L. Medicinal mushrooms. Evid Based Complement Alternat Med. 2014;2014:806180.
11. da Silva AF, Sartori D, Macedo Jr FC, Ribeiro LR, Fungaro MH, et al. Effects of beta-glucan extracted from Agaricus blazei on the expression of ERCC5, CASP9, and CYP1A1 genes and metabolic profile in HepG2 cells. Hum Exp Toxicol. 2013:32(6):647-54.

12. Monro JA. Treatment of cancer with mushroom products. Arch Environ Health. 2003:58(8):533-7.

13. Jeong SC, Koyyalamudi SR, Hughes J, Khoo C, Bailey T, et al. Antioxidant and immunomodulating activities of exo-and endopolysaccharide fractions from submerged mycelia cultures of culinary-medicinal mushrooms. Int J Med Mushrooms. 2013;15(3):251-66.

14. Wang J, Liu YM, Cao W, Yao KW, Liu ZQ, et al. Anti-inflammation and antioxidant effect of Cordymin, a peptide purified from the medicinal mushroom Cordyceps sinensis, in middle cerebral artery occlusion-induced focal cerebral ischemia in rats. Metab Brain Dis. 2012;27(2):159-65.

15. Xu T, Beelman RB, Lambert JD. The cancer preventive effects of edible mushrooms. Anticancer Agents Med Chem. 2012;12(10):1255-63.

16. Walton EL. Buried treasure: unlocking the secrets of medicinal mushrooms. Biomed J. 2014;37(6):339-42.

17. Nagano M, Shimizu K, Kondo R, Hayashi C, Sato D, et al. Reduction of depression and anxiety by 4 weeks Hericium erinaceus intake. Biomed Res. 2010;31(4):231-7.

18. Cui J, Chisti Y. Polysaccharopeptides of Coriolus versicolor: physiological activity, uses, and production. Biotechnol Adv. 2003;21(2):109-22.

19. Joshi YB, Pratico D. The 5-lipoxygenase pathway: oxidative and inflammatory contributions to the Alzheimer's disease phenotype. Front Cell Neurosci. 2014;8:436

20. Wu J, Wang A, Min Z, Xiong Y, Yan Q, et al. Lipoxin A4 inhibits the production of proinflammatory cytokines induced by beta-amyloid in vitro and in vivo. Biochem Biophys Res Commun. 2011;408(3):382-7.

21. McGeer PL, McGeer EG. Innate immunity, local inflammation, and degenerative disease. Sci Aging Knowledge Environ. 2002;2002(29):re3.

22. Hawkins KE, DeMars KM, Singh J, Yang C, Cho HS, et al. Neurovascular protection by post-ischemic intravenous injections of the lipoxin A4 receptor agonist, BML-111, in a rat model of ischemic stroke. J Neurochem. 2014;129(1):130-42.

23. Yang $F$, Xie J, Wang $W$, Xie $Y$, Sun $H$, et al. Regional arterial infusion with lipoxin A4 attenuates experimental severe acute pancreatitis. PLoS One. 2014:9(9):e108525.

24. Trovato A, Siracusa R, Di Paola R, Scuto M, Fronte V, et al. Redox modulation of cellular stress response and lipoxin A4 expression by Coriolus versicolor in rat brain: Relevance to Alzheimer's disease pathogenesis. Neurotoxicology, 2015. doi: 10.1016/j.neuro.2015.09.012

25. Taylor JP, Hardy J, Fischbeck KH. Toxic proteins in neurodegenerative disease. Science. 2002;296(5575):1991-5.

26. Buratti L, Balestrini S, Altamura C, Viticchi G, Falsetti L, et al. Markers for the risk of progression from mild cognitive impairment to Alzheimer's disease. J Alzheimers Dis. 2015:45(3):883-90.

27. Cornelius C, Trovato Salinaro A, Scuto M, Fronte V, Cambria MT, et al. Cellular stress response, sirtuins and UCP proteins in Alzheimer disease: role of vitagenes. Immun Ageing. 2013;10(1):41.

28. Castello MA, Soriano S. On the origin of Alzheimer's disease. Trials and tribulations of the amyloid hypothesis. Ageing Res Rev. 2014;13:10-2.

29. Sulistio YA and Heese K. The Ubiquitin-Proteasome System and Molecular Chaperone Deregulation in Alzheimer's Disease. Mol Neurobiol. 2015. doi: 10.1007/s12035-014-9063-4

30. Dunn HC, Ager RR, Baglietto-Vargas D, Cheng D, Kitazawa M, et al. Restoration of lipoxin A4 signaling reduces Alzheimer's disease-like pathology in the 3xTgAD mouse model. J Alzheimers Dis. 2015;43(3):893-903.

31. Abdelmoaty S, Wigerblad G, Bas DB, Codeluppi S, Fernandez-Zafra T, et al. Spinal actions of lipoxin A4 and 17(R)-resolvin D1 attenuate inflammation-induced mechanical hypersensitivity and spinal TNF release. PLoS One. 2013;8(9):e75543.

32. Jo Feeney M, Miller AM, Roupas P. Mushrooms-biologically distinct and nutritionally unique: exploring a "Third Food Kingdom". Nutr Today. 2014; 49(6):301-7.

33. Uhl GR. Hypothesis: the role of dopaminergic transporters in selective vulnerability of cells in Parkinson's disease. Ann Neurol. 1998;43(5):555-60.

34. Junn E, Mouradian MM. Apoptotic signaling in dopamine-induced cell death: the role of oxidative stress, p38 mitogen-activated protein kinase, cytochrome c and caspases. J Neurochem. 2001;78(2):374-83.

35. Calabrese V, Fariello RG. Regional distribution of malonaldehyde in mouse brain. Biochem Pharmacol. 1988;37(11):2287-8. 
36. Calabrese V, Mancuso C, Ravagna A, Perluigi M, Cini C, et al. In vivo induction of heat shock proteins in the substantia nigra following L-DOPA administration is associated with increased activity of mitochondrial complex I and nitrosative stress in rats: regulation by glutathione redox state. J Neurochem. 2007;101(3):709-17.

37. Castellani RJ, Rolston RK, Smith MA. Alzheimer disease. Dis Mon. 2010;56(9): 484-546.

38. Mori K, Obara Y, Moriya T, Inatomi S, Nakahata N. Effects of Hericium erinaceus on amyloid beta(25-35) peptide-induced learning and memory deficits in mice. Biomed Res. 2011;32(1):67-72.

39. Mori K, Ouchi K, Hirasawa N. The Anti-Inflammatory Effects of Lion's Mane Culinary-Medicinal Mushroom, Hericium erinaceus (Higher Basidiomycetes) in a Coculture System of 3 T3-L1 Adipocytes and RAW264 Macrophages. Int J Med Mushrooms. 2015;17(7):609-18.

40. Mori K, Inatomi S, Ouchi K, Azumi Y, Tuchida T. Improving effects of the mushroom Yamabushitake (Hericium erinaceus) on mild cognitive impairment: a double-blind placebo-controlled clinical trial. Phytother Res. 2009;23(3):367-72.

41. Lin PY, Simon SM, Koh WK, Folorunso O, Umbaugh CS, et al. Heat shock factor 1 over-expression protects against exposure of hydrophobic residues on mutant SOD1 and early mortality in a mouse model of amyotrophic lateral sclerosis. Mol Neurodegener. 2013:8:43.

42. Calabrese V, Dattilo S, Petralia A, Parenti R, Pennisi M, et al. Analytical approaches to the diagnosis and treatment of aging and aging-related disease: redox status and proteomics. Free Radic Res. 2015;49(5):511-24.

43. Dattilo S, Mancuso C, Koverech G, Di Mauro P, Ontario ML, et al. Heat shock proteins and hormesis in the diagnosis and treatment of neurodegenerative diseases. Immun Ageing. 2015;12:20.

44. Calabrese V, Cornelius C, Dinkova-Kostova AT, Calabrese EJ, Mattson MP. Cellular stress responses, the hormesis paradigm, and vitagenes: novel targets for therapeutic intervention in neurodegenerative disorders. Antioxid Redox Signal. 2010;13(11):1763-811.

\section{Submit your next manuscript to BioMed Central and we will help you at every step:}

- We accept pre-submission inquiries

- Our selector tool helps you to find the most relevant journal

- We provide round the clock customer support

- Convenient online submission

- Thorough peer review

- Inclusion in PubMed and all major indexing services

- Maximum visibility for your research

Submit your manuscript at www.biomedcentral.com/submit 